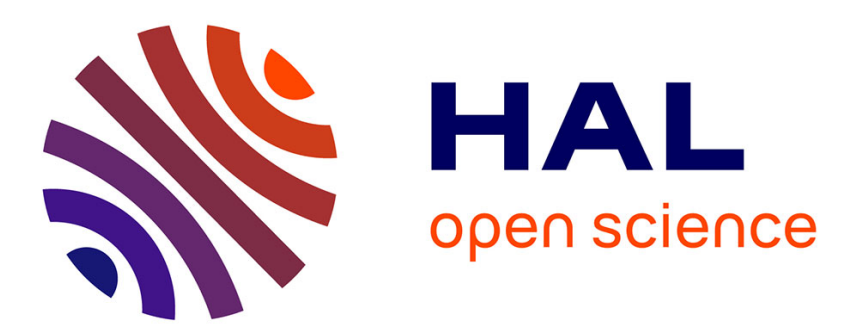

\title{
Influence of mechanical noise inside a scanning electron microscope.
}

Marcelo Gaudenzi de Faria, Yassine Haddab, Yann Le Gorrec, Philippe Lutz

\section{To cite this version:}

Marcelo Gaudenzi de Faria, Yassine Haddab, Yann Le Gorrec, Philippe Lutz. Influence of mechanical noise inside a scanning electron microscope.. Review of Scientific Instruments, 2015, 86 (4), pp.045105. 10.1063/1.4917557 . hal-01303490

\section{HAL Id: hal-01303490 \\ https://hal.science/hal-01303490}

Submitted on 18 Apr 2016

HAL is a multi-disciplinary open access archive for the deposit and dissemination of scientific research documents, whether they are published or not. The documents may come from teaching and research institutions in France or abroad, or from public or private research centers.
L'archive ouverte pluridisciplinaire HAL, est destinée au dépôt et à la diffusion de documents scientifiques de niveau recherche, publiés ou non, émanant des établissements d'enseignement et de recherche français ou étrangers, des laboratoires publics ou privés. 


\title{
Influence of mechanical noise inside a scanning electron microscope
}

\author{
Marcelo Gaudenzi de Faria, 1, a) Yassine Haddab, 1, b) Yann Le Gorrec, ${ }^{1,(\text { ) })}$ and Philippe Lutz ${ }^{1, d)}$ \\ FEMTO-ST, Besançon, France
}

The scanning electron microscope is becoming a popular tool to perform tasks that require positioning, manipulation, characterization and assembly of micro components. However, some of867iuj those applications require a higher level of dynamic accuracy and precision than those offered by available methods. Indeed, one limiting factor for the performance is the presence of unidentified noises and disturbances. This work aims to study the influence of mechanical disturbances generated by the environment and by the microscope, identifying how those can affect elements in the vacuum chamber. For that a dedicated setup, including a highresolution vibrometer, was built inside the microscope. This work led to the identification and quantification of the main disturbances and noise sources. Furthermore, the effects of external acoustic excitation were analysed. Potential applications of this results include noise compensation and real-time control for high accuracy tasks.

\section{INTRODUCTION}

Scanning electron microscopes (SEM) are tools with growing popularity when performing micromanipulation tasks, as they offers high-resolution images in a controlled environmental condition. They are particularly useful when high accuracies are needed. Applications include accurate positioning of components, force measurement, sample characterization and assembling tasks, and can have implications in several fields (material science, biology and medicine, physics, ...), allowing new functionalities and lowering their costs 12 .

The challenge of micro and nano-manipulation can be illustrated trough a comparison with the classical (macroworld) robotic manipulation tasks. While a typical industrial robot (with dimensions around $10^{\circ} \mathrm{m}$ ) can achieve position accuracies in the range of millimetres $\left(10^{-3} \mathrm{~m}\right)$, dedicated robots for micro-manipulation have dimensions close to decimetre $\left(10^{-1} \mathrm{~m}\right)$ and are able to achieve accuracies from hundreds of nanometers to a few nanometers for positioning tasks $\left(10^{-7}-10^{-9} \mathrm{~m}\right), \frac{3}{3}$. In order to develop high-performance automated tasks, the elements that can degrade the overall system's performance when executing micro-manipulation tasks should be considered:

- Difficulties in determination of the object state (positions, velocities, temperature, ...) and and uncertainties in physical parameter identification (mass, damping, ...). Available sensors are generally too large to be easily integrated, and the resolution requires are often at the limits of their capabilities.

- External disturbance sources. Effects that could be disregarded when working in the macro-world may dominate the behaviour of a micro device. The vibration produced by a machine, environmental

\footnotetext{
a) Electronic mail: marcelo.gaudenzi@femto-st.fr

b) Electronic mail: yassine.haddab@femto-st.fr

c) Electronic mail: legorrec@femto-st.fr

d) Electronic mail: philippe.lutz@femto-st.fr
}

changes (pressure, temperature variation, ...), and acoustic effects may contain enough energy to disturb the system.

Authors working in micro-manipulation in $\mathrm{SEM} \mathrm{s}^{3 / 46}$ 11 resort to embedded sensors and imaging feedback to perform the tasks. While end-effectors can be statically positioned with accuracies in the order of a few nanometers through the usage of filters (i.e averaging algorithms, statistical methods, ...), those methods are not adapted for dynamic performance, as they require longer/multiple acquisitions.

Therefore, the system's dynamic is often disregarded and the positioning task is approximated. As those dynamic effects may be not meaningful for many operations in the micrometre scale, they become an important limiting factor when we move towards nanometric precision. An example of how dynamic disturbances may limit the system's performance can be found in Kim et al. ${ }^{9}$, where a piezoelectric motor is used to drive a manipulating arm inside a field-emission SEM. While their actuator's nominal step size can be adjusted as low as $1 \mathrm{~nm}$, the practical least increment observed was in the range of $10 \mathrm{~nm}$ despite the vacuum environment. The source for this difference was finally credited to surrounding vibrations.

This work aims to study the dynamic behaviour of components inside a SEM, quantifying the effects of different disturbance sources and how they can degrade tasks in this environment. This information can be used to improve the behaviour of manipulation systems inside SEM and to correct disturbance effects, moving towards real-time control strategies with high accuracy and dynamic performances.

The paper is divided into four parts: Section II exposes the importance of SEM as a platform for micromanipulation, and how disturbances can affect its behaviour. It also describes how vacuum can affect beams. Section III explains the proposed experimental setup, the calibration of the sensor, and shows how the different pressures affect the samples. Section IV]describes the experimental steps followed to identify the different disturbance sources and how external acoustic sources can affect elements inside the vacuum chamber. Finally, section $\mathrm{V}$ presents our 
conclusions and considerations.

\section{SCANNING ELECTRON MICROSCOPE AS A PLATFORM FOR MANIPULATION}

A useful tool for automating the handling of parts and control of manipulators in the micro world is the use of imaging feedback. The SEM offers a controlled environment and produces topographic images by the sequential point-by-point bombardment and detection of radiation elements emitted by the sample, with resolution close to 1 nanometre. Those images are then used in positioning tasks and feature measurements. However, to improve their quality and signal/noise ratio, the averaging of several measurements is often required, resulting in acquisition times ranging from 0.1 up to hundreds of seconds.

\section{A. Noise in SEM}

Much of the research focusing on SEM noise is given to its imaging components, where aspects such as mechanical vibration and electromagnetic fields cause degradation in the image's quality $12-15$. However, disturbances can also affect physical components inside the SEM's chamber (samples, manipulators, supports, ...).

Mechanical disturbances can affect the microscope through the floor and through the air (acoustic vibrations). Figure 1 shows how those elements interact with the SEM. Ground vibrations from the surroundings (red) are transmitted to the SEM and components in its interior through mechanical coupling of the base. Microscopes are normally equipped with dampers to reduce the influence of ground vibrations. Acoustic waves disturbances collide with the external walls of the microscope, producing displacements in the electron column and deteriorating the image. Aware of those problems, the manufacturers indicate limits for ground and acoustic noise in the SEM room to acquire high-quality images. It is known that ground or acoustic vibrations can cause blur in images due to mechanical transmission, despite the use of vibration suppressors in the microscope and vacuum pumps for disturbance reduction. However, little is known about how those disturbances act over components inside the specimen chamber. Quantifying how those mechanical disturbances can be transmitted to the SEM's interior and affect samples and other components is an important step.

Vibration measurement and its frequency analysis provide a tool to detect and quantify the noise sources. Once those sources are identified, possible solutions include the suppression of the source (turning off unnecessary equipment, changing for a low-noise instrument...), the passive reduction of their effects (dampers, improving the acoustics of the room, ...) or the active reduction/rejection of their effects, through the developments

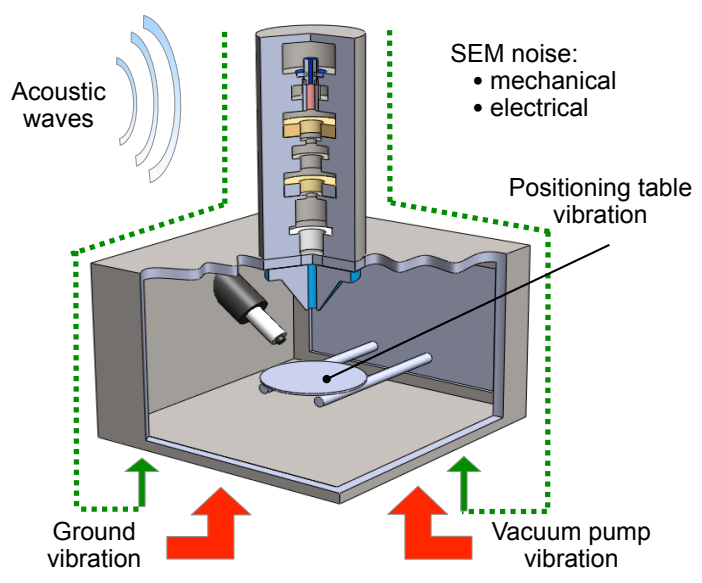

Figure 1: Expected sources of disturbance on the electron microscope.

of fast, dynamic systems capable of real-time compensations.

\section{B. Vacuum effects on beams}

When operating micro-components in the vacuum environment of a SEM, the effects of pressure variation on the samples should be considered. It is important to analyse how this variation can change the dynamical behaviour of MEMS components such as cantilevers and membranes, as the environment pressure affects their resonant modes frequencies and damping.

It is possible to distinct environmental conditions (flow regimes) due to pressure variations $\frac{16}{5}$. The flow regimes are defined by the Knudsen number $K_{n}$ :

$$
K_{n}=\frac{\lambda}{w}=\frac{1}{D \sigma w}
$$

where $\lambda$ is the mean free path of the gas molecule, $w$ the width of the gas layer motion, $D$ the gas number density and $\sigma$ the collision cross section of the gas. Three different flow regimes can be discerned: free molecule regime $\left(K_{n}>10\right)$, transition regime $\left(10>K_{n}>0.01\right)$ and viscous regime $\left(K_{n}<0.01\right)$. In the free molecular regime, the fluid slips with respect to the cantilever surface and the damping is proportional to the cantilever velocity. For the viscous regime, the gas properties are mainly governed by molecule-molecule collisions, and the cantilever acceleration becomes the important factor, inducing an increase in the effective mass of the cantilever. The changes in the frequency and damping between atmospheric pressure and vacuum depends on the geometry and the material composition of the part.

One analytical expression for estimating the change in modes on a cantilever of thickness $t$ and width $w$ due to 
variation in pressure was given by Lindholm in 1965 and used in 17 :

$$
f_{\text {gas }}=f_{v a c}\left(1+\frac{\pi M p w}{4 R T \rho t}\right)^{-1 / 2}
$$

with $M$ the molar mass of the gas, $p$ the environment pressure, $R$ the gas constant and $T$ the absolute temperature. For a $i$-layers cantilever, $\rho t$ should be replaced by $\sum \rho_{i} t_{i}$. Experimental results shown a reasonable agreement with the theory 17 , as the estimation of parameters for micro and nano- components is still a difficulty. Nevertheless, the frequency shift due to pressure variations is small $(2.5 \%$ or less $)$ in the studied references 17.20 .

There are several mechanisms contributing to the damping of oscillating cantilevers 21 . The total damping, or the inverse of the effective quality-factor (also called Q-factor) $1 / Q_{\text {eff }}$, can be defined as the sum of different factors: the intrinsic damping $1 / Q_{0}$, the fixation damping $1 / Q_{\text {mount }}$ and the air damping $1 / Q_{\text {air }}$.

$$
\frac{1}{Q_{e f f}}=\frac{1}{Q_{0}}+\frac{1}{Q_{\text {mount }}}+\frac{1}{Q_{\text {air }}}
$$

The intrinsic damping $1 / Q_{0}$ may be further partitioned as a sum of its major contributions: volume loss $1 / Q_{\text {loss }}$, support loss $1 / Q_{\text {support }}$, thermoelastic damping $1 / Q_{T E D}$ and surface loss $1 / Q_{\text {surf }}$. The effective Q-factor cannot exceed the value of the smallest $Q$ contribution. As the determination of some of those elements can become a difficult task, the determination of the most important mechanism is often enough for practical purposes. The damping factor of cantilevers can change by factors of $10^{4}$, although should be constant when working in the molecular free regime, what usually occurs in pressures below $10^{-2}$ mbar. In this region, intrinsic and fixation damping become the dominant mechanism for energy dissipation 18 . It is worth to remark that non-linearities, such as the jump phenomenon and harmonics, can occur on oscillating components $\frac{1922}{24}$ and may be more evident when working on vacuum. The jump phenomenon induces discontinuous system's response on forced systems due to the presence of multiple stable solutions, while harmonic non-linearity occurs when systems response contain frequencies other than the forcing frequency.

\section{EXPERIMENTAL SETUP}

To be able to measure the displacements inside a SEM and to allow noise analysis and real-time compensation, the proposed method uses a dedicated vibrometer instead of imaging techniques, such as the stroboscopic electron scanning microscopy $25 \mid 26$, or techniques that use the electron beam to measure displacements 27 . The use of a vibrometer allows to obtain both sub-nanometric resolution with a wide bandwidth, up to hundreds of $\mathrm{kHz}$.
The setup consists on fixing the vibrometer inside the SEM (Carl Zeiss SEM Auriga 60, shown in Figure 2) at a $45^{\circ}$ angle, in a way that both the laser and the electron beam intersect. This allows to acquire images and perform displacement measurements simultaneously. The sample is positioned using the SEM's table, what is necessary to adjust the laser beam incident angle. The support for the vibrometer is fixed to the SEM's door, allowing it to capture movements on the microscope positioning table with a minimum interference. Finally, the sensor is connected to its external box through the appropriate feed-though ports (optical fibre and electrical connection), allowing real time data acquisition. Figure 3 shows the scheme for this setup, and its practical implementation, where the vibrometer is attached to the SEM's door though an aluminium support.

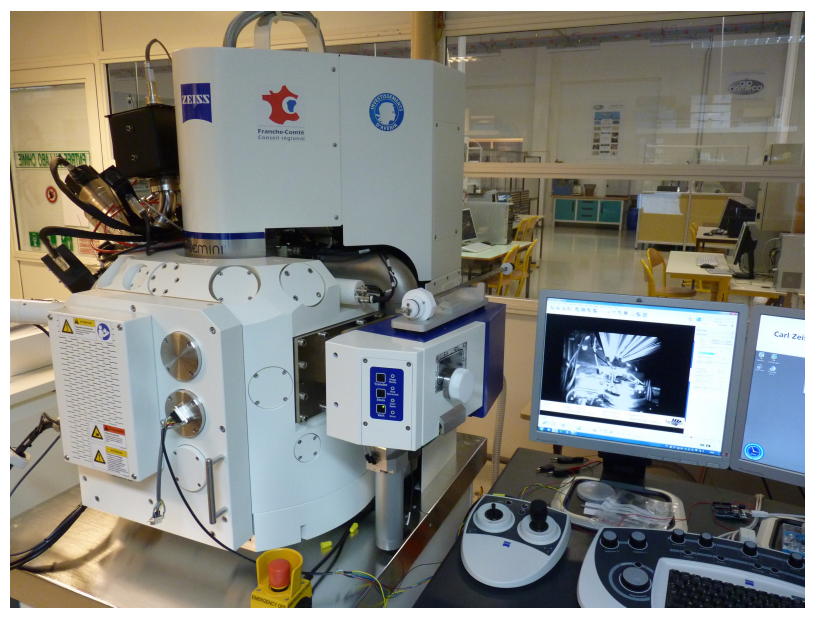

Figure 2: Carl Zeiss SEM Auriga 60, from the EQUIPEX ROBOTEX project, where the experiments were performed.

\section{A. Vibrometer characterization}

In order to estimate the disturbance levels in the system, it is necessary to quantify what are the sensor's intrinsic noise levels. This is obtained by measuring the vibration of a rigid metal block. Both the block and the vibrometer are fixed over a pneumatic anti-vibration table, and several measurements were performed for different reflection levels. The reflection level indicates the laser beam percentage that is reflected back by the sample and captured by the sensor and can be interpreted as the signal quality, where a higher value ensures a lower noise level. The nominal diameter of the laser spot on focus is $12 \mu \mathrm{m}$.

The measurements for the calibration tests were performed with an acquisition frequency of $12.5 \mathrm{kHz}$. For each different reflection level considered for the calibration process $(23,35,40,50,65 \%)$, a series of 10 measurements were performed and the resulting signal was pro- 
(a)

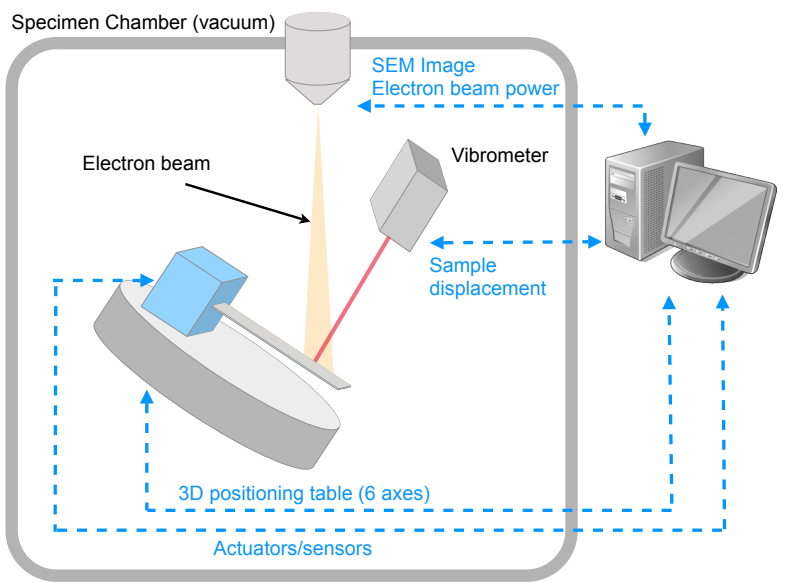

(b)

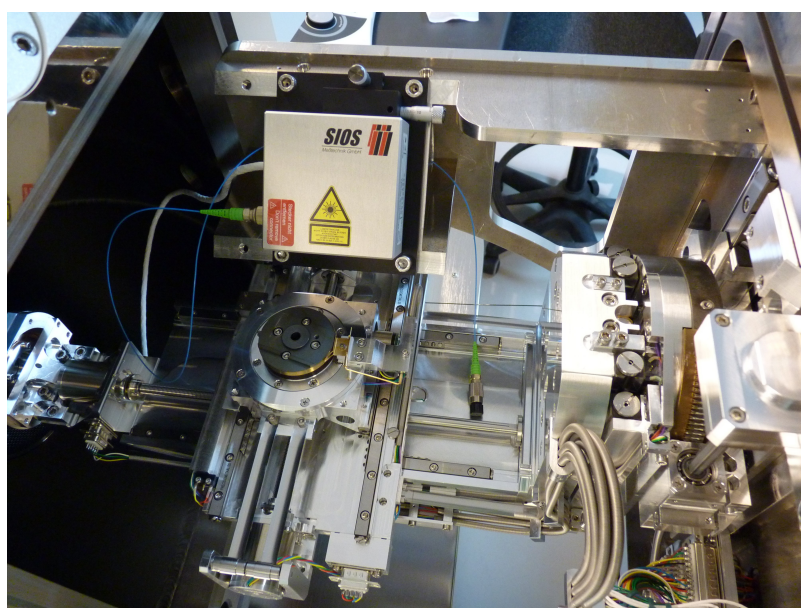

Figure 3: Scheme of the experimental setup proposed (a) and implementation inside the SEM (b).

cessed using the Welch power spectrum density method. Those 10 resulting frequency spectra were averaged to obtain the estimation of the sensors' frequency response. The results presented in Figure 4, confirm that noise levels are related to the reflection levels, showing the characteristic spectrum of white noise, except for peaks around 800 and $4000 \mathrm{~Hz}$. While the first have small magnitude and is apparent only at high reflection levels, the second is present in all measurements and have an important component. The average RMS noise level is computed for the different conditions, indicating that subnano-metric accuracy can be achieved with this device for reflections over $45 \%$.

\section{B. Influence of pressure on the dynamic response}

Before starting the identification of disturbances on the SEM, experiments were performed with the samples at (a)

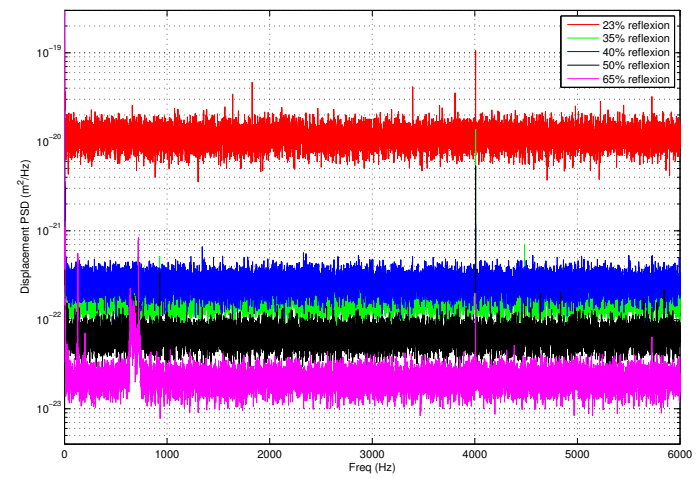

(b)

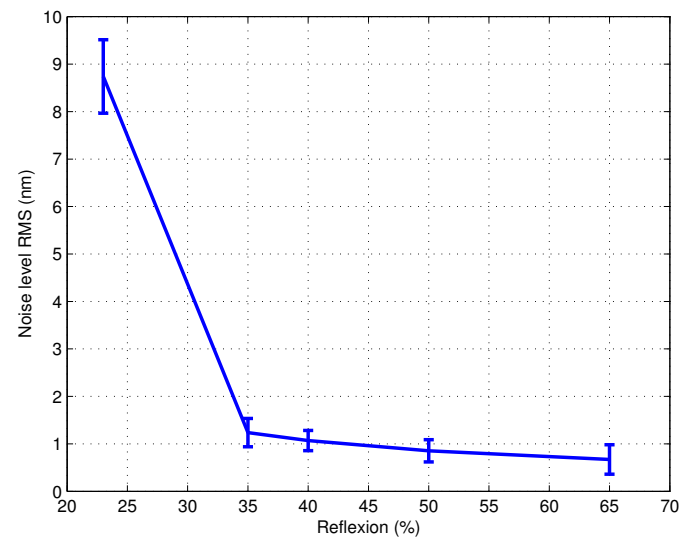

Figure 4: Results for the vibrometer calibration. (a) shows the PSD for different reflection values, from $23 \%$ to $65 \%$. (b) shows the average RMS value of noise for each reflection percentage, together with its standard deviation.

atmospheric pressure and high vacuum to observe and compare the differences between both cases. The tests aimed to obtain the characteristics of a set of silicon cantilevers of different lengths $L$ and also a commercial micro-gripper. The samples were fixed in supports over the positioning table inside the SEM, where they were test for both pressure conditions.

Figure 5 shows the silicon sample fixed in the holder at 45 degrees. The measurements inside the microscope were performed with both pressures, in the range of $9.10^{-7}$ to $4.10^{-6}$ mbar for vacuum (operating pressure for the microscope's chamber) and at atmospheric pressure. Working at this vacuum levels ensure that the system operates in the free molecular regime $\left(K_{n}>10\right)$. Also no electron beam bombardment was applied during tests. The laser beam was aimed at the cantilever's tip, with reflections between 45 and $82 \%$. The second sample was the SEM compatible micro-gripper FTG-30 from the Swiss enterprise Femtotools (Figure 6). Its left finger possesses an embedded electrostatic comb-drive, 


\begin{tabular}{lcc}
\hline \hline Name & Dimensions $(\mathrm{l} \times \mathrm{w} \times \mathrm{t}, \mathrm{in} \mu \mathrm{m})$ & $f_{1_{\text {air }}}(\mathrm{Hz})$ \\
\hline Cantilever 1 & $5000 \times 400 \times 20$ & 1162.0 \\
Cantilever 2 & $4000 \times 400 \times 20$ & 2013.4 \\
Cantilever 3 & $3000 \times 400 \times 20$ & 3649.1 \\
Cantilever 4 & $2000 \times 400 \times 20$ & 8337.4 \\
Micro-gripper ${ }^{\natural}$ & $4000 \times 120 \times 50$ & 1122.3 \\
\hline \hline
\end{tabular}

${ }^{\text {a }}$ Due to its complex geometry, values are approximated

Table I: Samples' dimensions (length $l$, width $w$ and thickness $t$ and first resonance mode (experimental) measured at atmospheric pressure.

while the right finger has a capacitive force sensor. During the measurements for this sample, the laser beam was pointed at the extremity of the left (actuated) finger with reflections between 45 and $60 \%$. Its first mode $(1122.3 \mathrm{~Hz}$ for a zero input voltage) is due to the comb-drive actuating system, and not the vibration mode of the finger itsel $^{28}$. Table I resumes the different sample's geometric characteristics and first modes observed at atmospheric pressure.

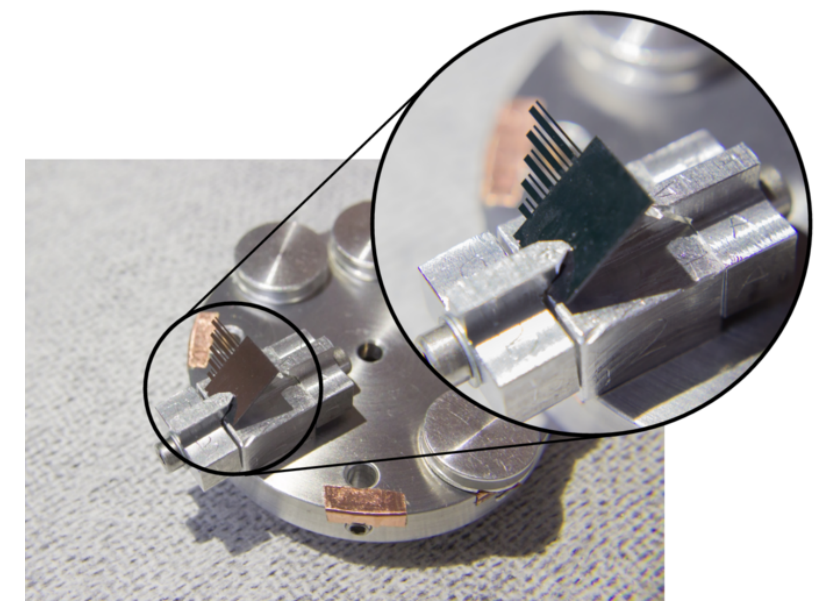

Figure 5: Sample holder containing the silicon cantilevers.

Applying Equation 2 allows to estimate the variation in frequencies for the first modes. Considering the molar mass of air $M=28.97 \mathrm{~g} \mathrm{~mol}^{-1}$, the atmospheric pressure $p=101325 \mathrm{~Pa}$, the gas constant $R=$ $8.314 \mathrm{~m}^{3} \cdot \mathrm{Pa} \cdot \mathrm{K}^{-1} \cdot \mathrm{mol}^{-1}$, the temperature $T=294.15 \mathrm{~K}$ (room temperature, 21 degrees Celsius), the silicon density $\rho=2329 \mathrm{Kg} \cdot \mathrm{m}^{-1}$, cantilever width $w=400 \mu \mathrm{m}$ and thickness $h=20 \mu \mathrm{m}$, is possible estimate the frequency shift due to the pressure variation. Matching the parameters unities, the nominal frequency variation of $\Delta f_{\text {theory }}$ of $0.4 \%$ from the atmospheric measured value is computed. The results are shown in table II The measured frequency agreed in different degrees with the theory, with important variations between the cantilevers. The parametric uncertainties can partially explain those

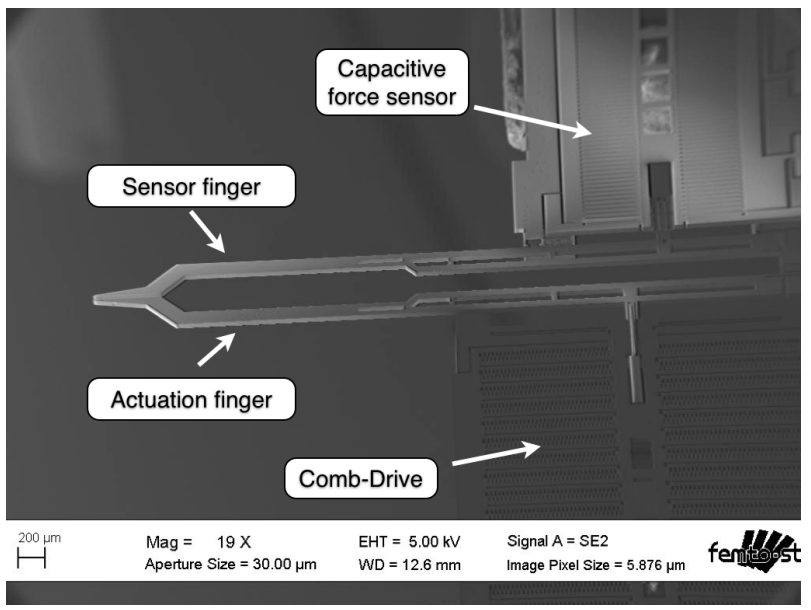

Figure 6: Micro-gripper FTG-30 (image acquired with SEM). The left finger can be actuated through a comb-drive mechanism.

\begin{tabular}{lcccc}
\hline \hline & \multicolumn{3}{c}{ Measured } & Estimated \\
\hline Name & $f_{1_{\text {air }}}$ & $f_{1_{\text {vac }}}$ & $\Delta f_{\text {meas }}(\%)$ & $f_{1_{\text {vac }}}$ \\
\hline Cantilever 1 & 1162.0 & 1169.6 & 0.65 & 1166.7 \\
Cantilever 2 & 2013.4 & 2022.9 & 0.47 & 2021.5 \\
Cantilever 3 & 3649.1 & 3664.4 & 0.42 & 3663.8 \\
Cantilever 4 & 8337.4 & 8366.8 & 0.35 & 8371.2 \\
MG (@ 25V) & 1066.7 & 1105.2 & 3.6 & - \\
\hline \hline
\end{tabular}

Table II: First resonance frequency (in $\mathrm{Hz}$ ) of different samples for both pressure conditions, and estimated resonance frequency in vacuum.

\section{differences.}

Applying the Equation 2 to the FTG-30 gripper parameters results in a theoretical frequency shift below $0.1 \%$, while experimental variations up to $3.6 \%$ were measured for its actuated arm. This difference in the values is due to the first mode of the gripper that is related to the comb-drive actuation system connected to the finger, and not to its beam characteristics, what renders the approximation equation non-valid for this case. Due to the complex geometry of the system consisting on combdrive plus finger, it becomes a harder task to analytically estimate this frequency. It is worth to mention that the micro-gripper modes depend on the finger's displacement. Therefore, for different operating points (input voltages), different values for the first mode are found.

To analyse variations in the damping factor, only the micro-gripper was employed, as its contains an actuation system. Applying voltage step of different values to it and measuring its dynamic responses at atmospheric pressure and inside the SEM, its Q-factor parameters were estimated. Figure 7 shows the time response for a $30 \mathrm{~V}$ step $(t=0)$ at atmospheric pressure and vacuum, and it is clear how the pressure have a great affect on this device's behaviour. To quantify this result, the assumptions of 


\begin{tabular}{lccc}
\hline \hline Name & $\frac{1}{Q_{\text {eff }}}($ air $)$ & $\frac{1}{Q_{\text {eff }}}($ vacuum $)$ & $\frac{1}{Q_{\text {air }}}($ estimated $)$ \\
\hline $10 \mathrm{~V}$ & $1.08 \cdot 10^{-2}$ & $1.91 \cdot 10^{-4}$ & $1.6 \cdot 10^{-2}$ \\
$15 \mathrm{~V}$ & $0.70 \cdot 10^{-2}$ & $1.64 \cdot 10^{-4}$ & $0.68 \cdot 10^{-2}$ \\
$20 \mathrm{~V}$ & $0.91 \cdot 10^{-2}$ & $1.92 \cdot 10^{-4}$ & $0.89 \cdot 10^{-2}$ \\
$25 \mathrm{~V}$ & $0.87 \cdot 10^{-2}$ & $1.92 \cdot 10^{-4}$ & $0.85 \cdot 10^{-2}$ \\
$30 \mathrm{~V}$ & $0.92 \cdot 10^{-2}$ & $2.21 \cdot 10^{-4}$ & $0.90 \cdot 10^{-2}$ \\
\hline
\end{tabular}

Table III: Micro-gripper quality factor variation for different operating points.

Equation 3 were applied to estimate the damping in both conditions. Table III resumes the obtained results. Applying different levels of excitation to the gripper, the quantified variation is of two orders of magnitude.

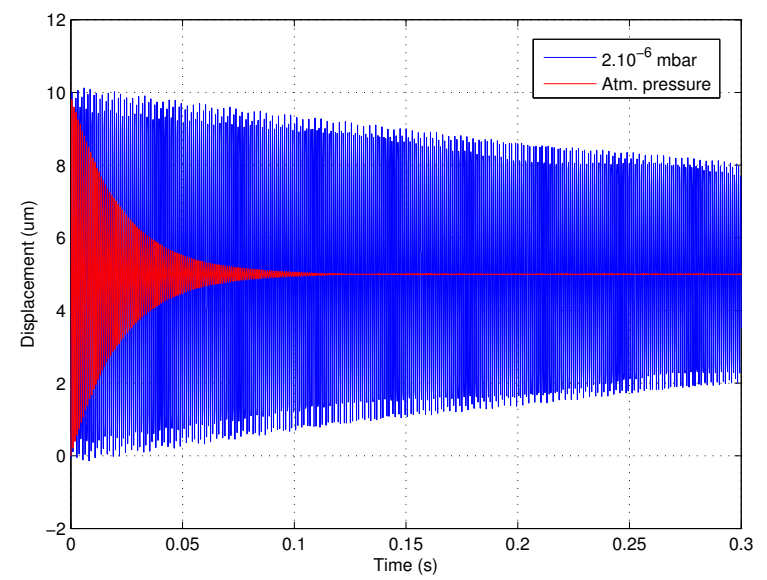

Figure 7: Comparison between micro-gripper's step responses for atmospheric pressure and vacuum for

$$
V_{\text {in }}=30 \mathrm{~V} \text {. }
$$

\section{NOISE AND DISTURBANCE CHARACTERIZATION}

To study the influence of external disturbances over components inside the SEM, two different tests have been performed. The first observed what are the perturbations constantly affecting the microscope under regular operation conditions. The second test a controlled external acoustic disturbance is added to observe its transmissibility to samples in the vacuum chamber.

\section{A. System under regular operation condition}

This step consisted on tests performed in high-vacuum conditions with various samples, aiming to identify the most important disturbances frequencies and to characterize its sources (mechanical, electrical, measurement noise). The silicon samples do not possess with any kind of actuators or electrical connections, and therefore all the displacements measures should be originated by mechanical vibrations (from inside and outside the SEM) transmitted to the cantilever, or sensor's noise. However, the micro-gripper should be subject to all three different noise sources.

The Welch's power density spectrum method was applied to the time domain data to obtain frequency information. For each cantilever, 20 measurements were performed and averaged in the frequency domain. This allows to filter sporadic disturbances that may had occurred during measures and further reduces measurement noise. Figure 8 shows the obtained curve for silicon cantilevers from 0 to $1 \mathrm{kHz}$. From the graph, six major peaks (56.1, 95.4, 140, 235.7, 688.55, and 955.2) in the spectrum frequency were identified, besides the cantilevers' resonant modes, as well as other minor peaks with small contributions to the spectrum. A higher frequency peak (4015.2 Hz and its harmonics) was also observed. Those values, not shown in Figure 8, are produced by the vibrometer (measurement noises).

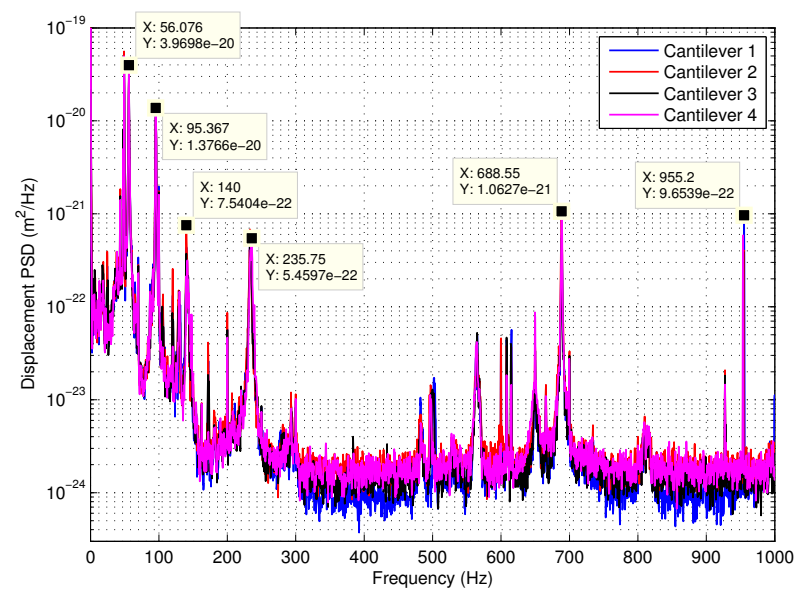

Figure 8: Averaged power spectrum density of the silicon cantilevers, detailing the frequencies with larger amplitude.

The RMS vibration level measured for the beam tips ranged from 1.07 to $2.55 \mathrm{~nm}$, while the measured displacements at the base of the cantilever showed RMS values between 0.86 and $0.98 \mathrm{~nm}$. Both tip and base showed similar frequency spectrum, with exception of the first cantilever's mode, responsible for the difference in the computed RMS value. The sample's first modes, with shifted frequency due do pressure variation, presents a large contribution to the total vibration, showing how the system can be still excited through mechanical coupling, even in a system that was believed to be isolated from the environment.

The two lower frequencies (56.1 and 95.4) were credited to the vibrometer's support. As this structure is fixed on the SEM's door, it can be seen as a clamped beam it- 
self. To verify this assumption, two tests were performed. The first consisted in changing the sensor's placement on the support. Positioning it closer to the support's base, those frequencies shifted to 82.3 and $122.5 \mathrm{~Hz}$, respectively, while other frequencies remain unchanged. The second test consisted in adding weights to the support and to the positioning table. Again, these frequencies shifted when the weights were on the vibrometer's support only, confirming this assumption. It is possible to conclude that those are measurement noise and do not represent actual displacements in the sample. Using the same principle, it was observed that the peaks located at 140 and $235.7 \mathrm{~Hz}$ changed their frequency when weights were placed on the positioning table, demonstrating that those frequencies are of mechanical origin. As it depends on the mass of the objects over the positioning table, those frequencies are not absolute.

To help identify the sources of the remaining peaks (954.8 and $697.2 \mathrm{~Hz}$ ), another experiments were performed considering two different situations:

To help identify the sources of the remaining frequency peak (954.8 and $697.2 \mathrm{~Hz}$ ), other experiments were performed considering two different situations:

- Condition 1: SEM in normal operation condition, with high vacuum $\left(10^{-6}\right.$ mbar $)$ and no electron beam bombardment.

- Condition 2: SEM powered off, with electricity shut off, with high vacuum $\left(10^{-6}\right.$ mbar $)$.

Enforcing Condition 2 ensures that SEM elements (i.e. vacuum pumps, cooling fans, ...) will be turned off, allowing to identify the contribution of those components as disturbance sources. As the specimen chamber stayed sealed, the vacuum level was kept in similar conditions as the other measurements. In Figure 9 the PSD for those frequencies on both cases are compared for Cantilever 1, and it is clear that the origin is related to the microscope. Similar results were found for other samples. The main frequency around $700 \mathrm{~Hz}$ is believed to be caused by the SEM's turbomolecular pump. Differently from the main rotary pump, this component is always active during SEM operation and have a nominal rotation frequency of $42000 \mathrm{rpm}$, in agreement with the experimental values. It is worth to remark that this pump is placed over a passive damping system and connected to the SEM using proper components to minimize the vibration.

The $954.8 \mathrm{~Hz}$ frequency also appears to be related to the SEM's operation. For a further analysis, the PSD of cantilevers and the micro-gripper around this frequency were compared, and the results are shown in Figure 10. The lower limits of the graph differ due to differences in the reflection level of the material. For different measures, performed in different days, shifts in this frequency occur. Repeating the experiment, it was noticed that those variations are time-dependent, and not related to the sample. However, in all experiments, this phenomenon was located between 950 and $956 \mathrm{~Hz}$. This effect was consistently more important for the microgripper than the silicon cantilevers, what could indicate components in electronic noise affecting both sensor and micro-gripper's electronic components.

(a) $700 \mathrm{~Hz}$

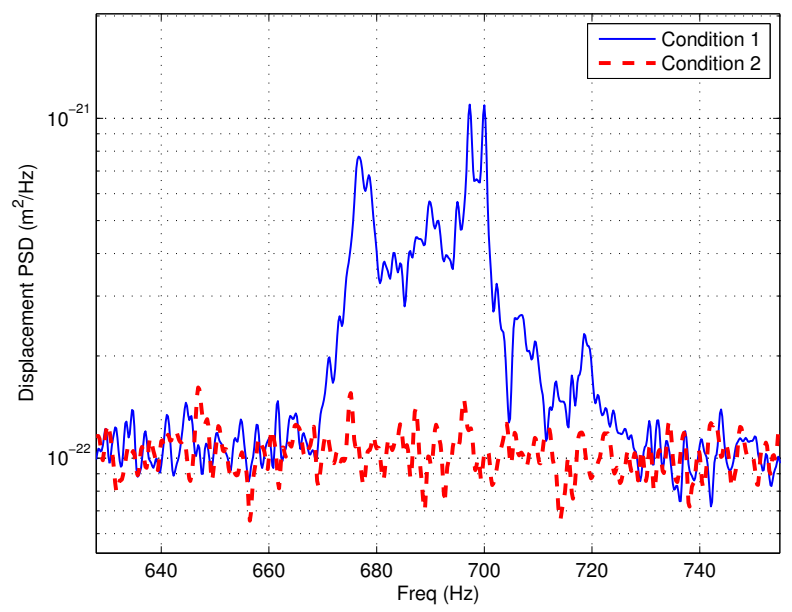

(b) $955 \mathrm{~Hz}$

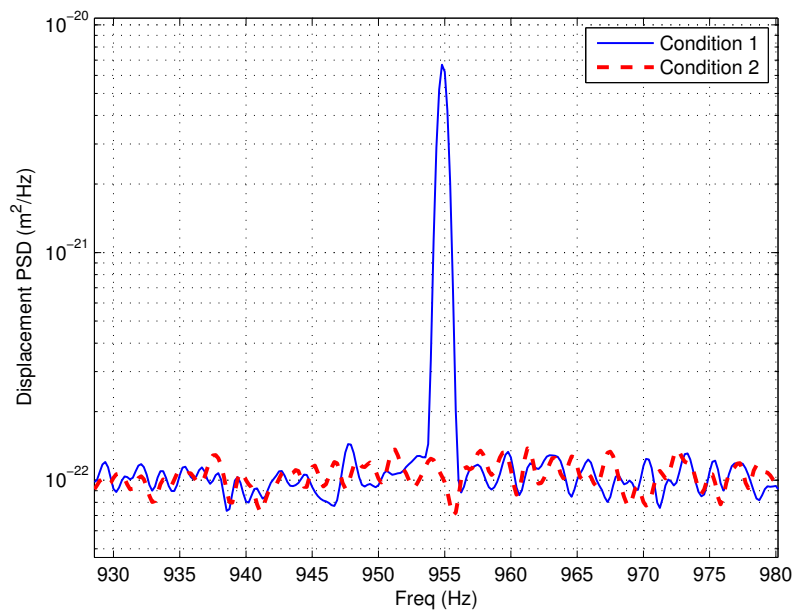

Figure 9: PSD around 700 (a) and $955 \mathrm{~Hz}$ (b) considering the SEM on two conditions, indicating that the SEM can be itself a source of disturbance.

Figure 11 shows how the vibration in the cantilever is reduced when the SEM is turned off. This demonstrates that the microscope can have a large contribution over disturbances. Other frequencies related to mechanical vibrations (56 and $95 \mathrm{~Hz}$ ) also showed decreased amplitudes, although they were still present in important levels. The average RMS displacement in this condition was reduced to $0.84 \mathrm{~nm}$, close to the measurement noise level.

The results found in this part can be summarized by Figure 12 showing the sources for each one of the most significant disturbances that were identified experiment- 


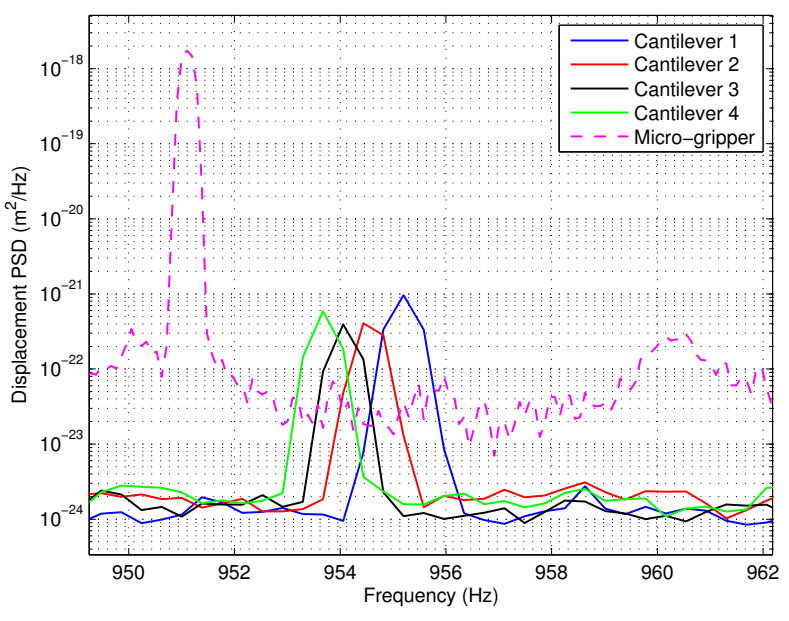

Figure 10: PSD around $955 \mathrm{~Hz}$ comparing silicon cantilevers and micro-gripper results.

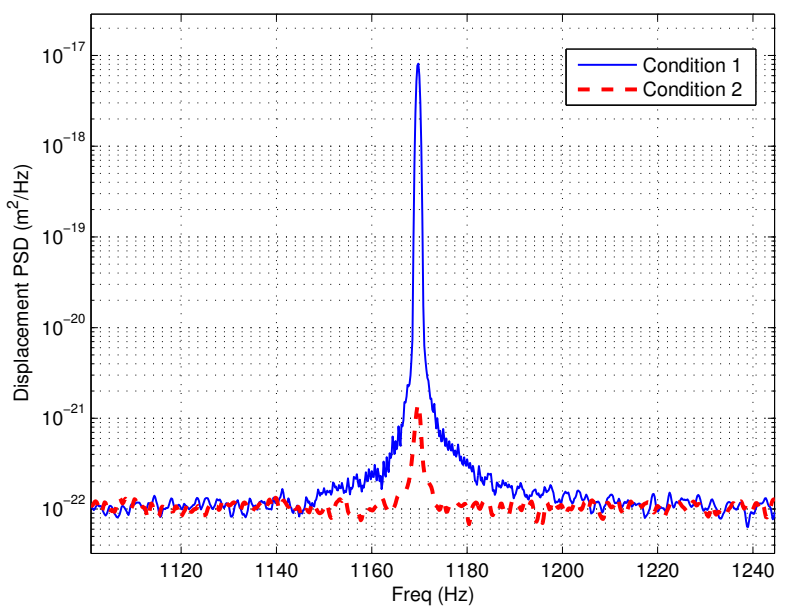

Figure 11: PSD of Cantilever 1, detailed for its first mode $(1169.8 \mathrm{~Hz})$, comparing both test conditions.

ally.

\section{B. System under external acoustic excitation}

In a second instance, the effects of an external acoustic excitation over samples in the vacuum chamber was tested. During the previous experiment, it was noticed that sounds produced near the SEM (i.e. speech in the room) had influence over the silicon cantilever sample. Through the use of a signal generator and a loud-speaker (constant frequency response from $1 \mathrm{kHz}$ to $10 \mathrm{kHz}$ ) positioned at 1 meter from the SEM, a controlled acoustic noise with different frequencies and amplitudes was generated, allowing to better verify this supposition.

A worst case scenario was tested, were the system was

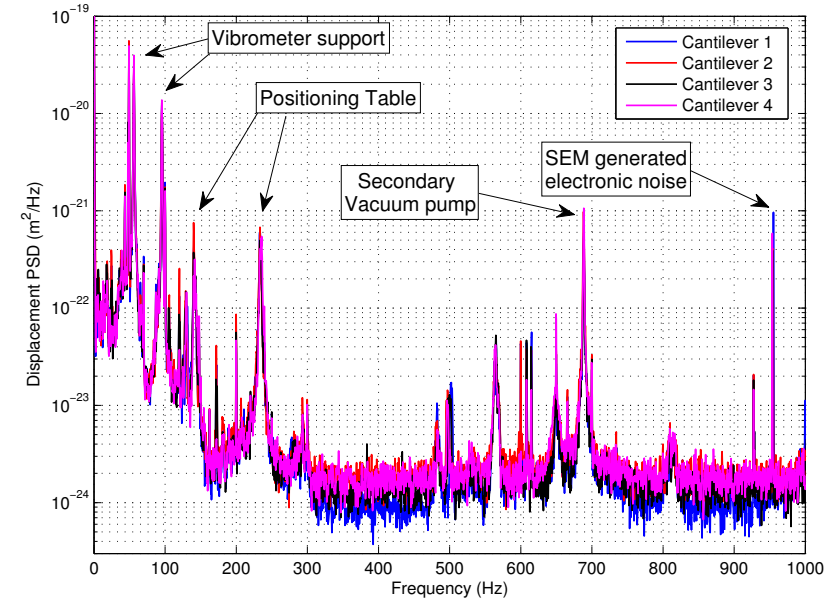

Figure 12: Resume of the most important measured sources of disturbance.

subjected to a constant sine wave disturbance. A set of sample displacement measurements was acquired before activating the loudspeaker, and the RMS displacement obtained for the beam was $1.07 \mathrm{~nm}$ (average of 10 measurements). Generating a sinusoidal signal of 1000 $\mathrm{Hz}$ thought the loudspeaker (outside the range of any resonant frequency previously measured), the displacements observed in the sample were small, only noticeable for high amplitudes (70 decibels of greater) and even so the effects were limited, as other mechanical disturbances previously measured were still dominant (RMS vibration level of $1.15 \mathrm{~nm}$ ). Nonetheless, if the frequency applied matches a resonant mode (1169.8 Hz in this case), much lower amplitudes can have important effects. For the Cantilever 1 , the system achieved a constant $21 \mathrm{~nm}$ peakto-peak vibration for an approximated excitation level of 46 decibels. Figure 13 exemplifies this effects.

The same experiment was performed using the microgripper. Figure 14 shows the time response of the system to different noise amplitudes (around 50 and $60 \mathrm{~dB}$ ) and frequency matching the first mode of the gripper. For comparison, the average level estimated for human conversation at 1 metre distance is of $60 \mathrm{~dB}$. It is clear that, even in a high vacuum environment, samples are still subject to acoustic disturbances through mechanical coupling.

While the scenario simulated in this experiment is unlikely to happen, a laboratory is prone to different acoustic disturbances and pressure variations from the environment that can affect one or multiple resonant modes of components in the specimen chamber, as their components can include large frequency bands of the spectrum. This test exemplifies how even low amplitude noise may have large impact on the positioning accuracy inside the SEM. 


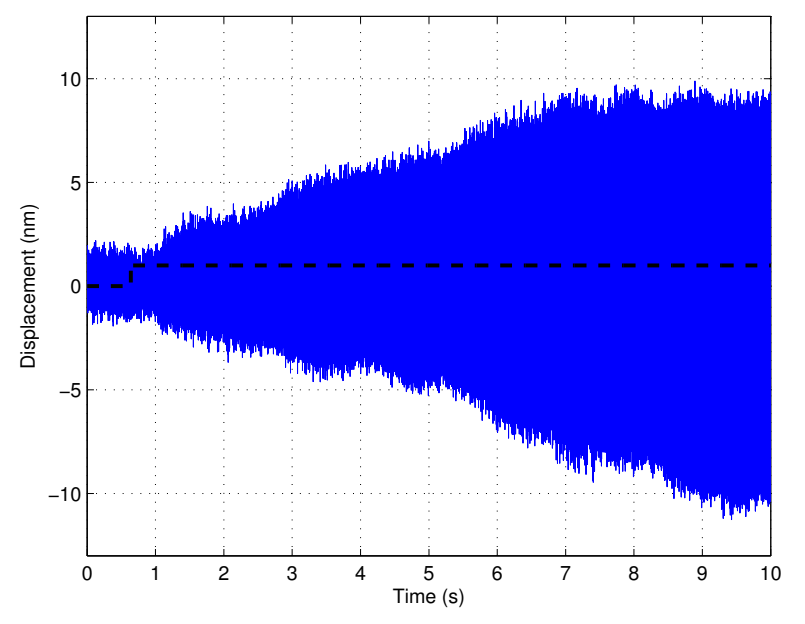

Figure 13: Displacement measurement of silicon cantilever under the influence of a external source of noise (loudspeaker). A sinusoidal wave with frequency matching the sample's first mode is applied. The black dotted line indicates when the external disturbance begins.

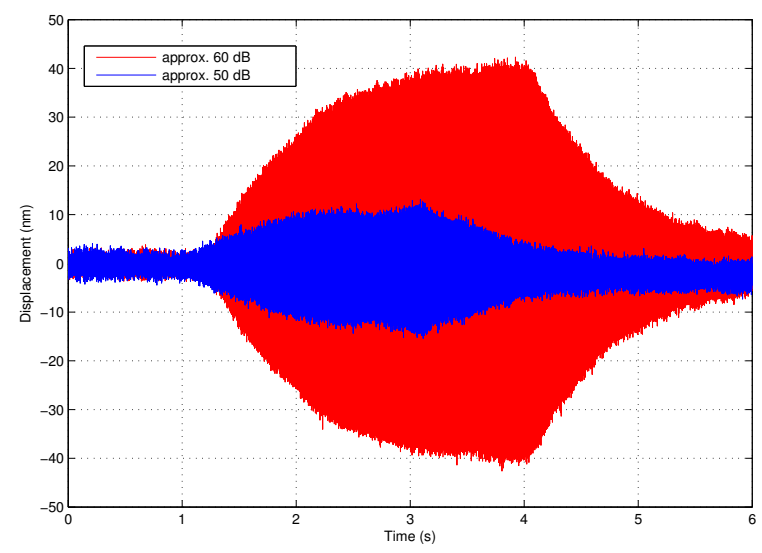

Figure 14: Micro-gripper displacement when subjected to external acoustic disturbances of different amplitudes.

\section{v. CONCLUSION}

In this paper, the most significative disturbances that can influence the manipulation in SEM were characterized. The proposed method was used to measure the vibration levels of different silicon cantilever and one microgripper, and allowed to identify the different disturbances acting over the samples. The measures were performed inside the specimen chamber of a SEM, with atmospheric and vacuum pressures, without the incidence of electron beams.

Despite being placed over an anti-vibration system, the microscope is still affected by its surrounding. Although the major displacement contributing frequency was the sample's first vibration mode, other elements ( SEM's positioning table and vacuum pump induced vibrations) can also contribute to the sample's vibration. Furthermore, the microscope itself was able to excite the sample's first mode during its operation, increasing the vibration amplitude. Another studied frequency, located around 955 $\mathrm{Hz}$, was shown to be generated by the microscope. Despite not being able to precisely identify its source, it is originated by the SEM and appears to be of electronic origin, affecting electronic components (sensor and electrical actuator) inside the vacuum chamber. A map of the existing noises was generated, where the most important sources could be identified. This information about the dynamic behaviour inside the chamber is a first step towards real time, dynamic noise rejection to achieve fast, precise manipulation and positioning inside a SEM.

It was demonstrated that acoustic disturbances can have influence on components inside the vacuum chamber as the vibrations propagate though the microscope's walls. In general, those vibrations had a small effects on samples. However as the acoustic noise frequency matches a vibration mode of the sample, the effect becomes more important, even for low power levels (46 dB), what could easily be found in a laboratory environment. The effect of acoustic pressure can be also noticed in everyday situations, i.e. conversations in the room, as the range of the human speech can contain the first mode of our samples. It is clear that special care should be taken when performing task with extreme precision requirements. Even weak pressure variations can have large effects on samples if a frequency composing the disturbance matches a resonant mode of a component inside the microscope.

\section{ACKNOWLEDGMENTS}

This work was supported in part by the French National Project NanoRobust under Grant ANR-2011NANO-006 and in part by the EQUIPEX ROBOTEX Project under Grant ANR-10-EQPX-44-01. The authors also would like to thank V. Petrini, J. Y. Rauch and the MIMENTO clean room team in the Femto-ST institute for the silicon cantilevers's fabrication.

\section{REFERENCES}

${ }^{1}$ P. Holister, "Nanotech: the tiny revolution," CMP Cientifica, Nov (2001).

${ }^{2}$ M. Schulenburg, "Nanotechnology: Innovation for tomorrow's world," (2004).

${ }^{3}$ S. Fatikow, Automated nanohandling by microrobots (Springer Publishing Company, Incorporated, 2007).

${ }^{4}$ D. Nakabayashi, P. Silva, and D. Ugarte, "Inexpensive two-tip nanomanipulator for a SEM," Applied Surface Science 254, 405411 (2007). 
${ }^{5}$ K. Y. Hwang, S.-D. Kim, Y.-W. Kim, and W.-R. Yu, "Mechanical characterization of nanofibers using a nanomanipulator and atomic force microscope cantilever in a scanning electron microscope," Polymer Testing 29, 375-380 (2010).

${ }^{6}$ S. Fahlbusch, S. Mazerolle, J.-M. Breguet, A. Steinecker, J. Agnus, R. Pérez, and J. Michler, "Nanomanipulation in a scanning electron microscope," Journal of materials processing technology 167, 371-382 (2005).

${ }^{7}$ Y. Zhang, Y. Zhang, C. Ru, B. Chen, and Y. Sun, "A loadlock-compatible nanomanipulation system for scanning electron microscope," ASME Transactions on Mechatronics 18 (2013).

${ }^{8}$ D. Jasper, SEM-based motion control for automated robotic nanohandling, Ph.D. thesis, Carl von Ossitezky Universität, Oldenburg (2011).

${ }^{9}$ K. Kim, S. Lim, I. Lee, K. An, D. Bae, S. Choi, J. Yoo, and Y. Lee, "In situ manipulation and characterizations using nanomanipulators inside a field emission-scanning electron microscope," Review of scientific instruments 74, 4021-4025 (2003).

${ }^{10}$ B. Polyakov, L. M. Dorogin, A. Lohmus, A. E. Romanov, and R. Lohmus, "In situ measurement of the kinetic friction of $\mathrm{ZnO}$ nanowires inside a scanning electron microscope," Applied Surface Science 258, 3227-3231 (2012).

${ }^{11}$ M. R. Roenbeck, X. Wei, A. M. Beese, M. Naraghi, A. Furmanchuk, J. T. Paci, G. C. Schatz, and H. D. Espinosa, "In situ scanning electron microscope peeling to quantify surface energy between multiwalled carbon nanotubes and graphene," ACS nano 8, 124-138 (2014).

${ }^{12}$ D. Muller, E. Kirkland, M. Thomas, J. Grazul, L. Fitting, and M. Weyland, "Room design for high-performance electron microscopy," Ultramicroscopy 106, 1033-1040 (2006).

${ }^{13} \mathrm{~A}$. Vladar, "Scanning electron microscopy in real world environment," (2003).

${ }^{14}$ K. O. Jung, S. J. Kim, and D. H. Kim, "An approach to reducing the distortion caused by vibration in scanning electron microscope images," Nuclear Instruments and Methods in Physics Research Section A: Accelerators, Spectrometers, Detectors and Associated Equipment 676, 5-17 (2012).

${ }^{15}$ M. Pluska, A. Czerwinski, J. Ratajczak, J. Katcki, and R. Rak, "Elimination of scanning electron microscopy image periodic distortions with digital signal-processing methods," Journal of microscopy 224, 89-92 (2006).

${ }^{16}$ J. Mertens, E. Finot, T. Thundat, A. Fabre, M.-H. Nadal,
V. Eyraud, and E. Bourillot, "Effects of temperature and pressure on microcantilever resonance response," Ultramicroscopy 97, 119-126 (2003).

${ }^{17}$ R. Sandberg, W. Svendsen, K. Mølhave, and A. Boisen, "Temperature and pressure dependence of resonance in multi-layer microcantilevers," Journal of Micromechanics and Microengineering 15, 1454 (2005).

${ }^{18}$ H. Sumali and T. G. Carne, "Air-drag damping on microcantilever beams," in XXVI International Modal Analysis Conference (IMAC): Conference ES Exposition on Structural Dynamics (Society of Experimental Mechanics, 2008).

${ }^{19}$ D. S. Epp, O. B. Ozdoganlar, and H. Sumali, "Dynamic measurement of gas damping effects in MEMS," in Current Proceedings, SEM International Congress $X$ (2004).

${ }^{20} \mathrm{~W}$. Zhang and K. L. Turner, "Pressure-dependent damping characteristics of microsilicon beam resonators for different resonant modes," in Sensors, 2005 IEEE (IEEE, 2005) pp. 4-pp.

${ }^{21}$ J. Lübbe, L. Tröger, S. Torbrügge, R. Bechstein, C. Richter, A. Kühnle, and M. Reichling, "Achieving high effective q-factors in ultra-high vacuum dynamic force microscopy," Measurement Science and Technology 21, 125501 (2010).

${ }^{22}$ C. M. Harris, A. G. Piersol, and T. L. Paez, Harris' shock and vibration handbook, Vol. 5 (McGraw-Hill New York, 2002).

${ }^{23}$ C. Anindya, "A brief introduction to nonlinear vibrations," Tech. Rep. (Indian Institute of Science, 2009).

${ }^{24}$ M. V. Requa and K. L. Turner, "Electromechanically driven and sensed parametric resonance in silicon microcantilevers," Applied Physics Letters 88, 263508 (2006).

${ }^{25}$ H. Fujioka, K. Nakamae, and K. Ura, "Function testing of bipolar ics and lsis with the stroboscopic scanning electron microscope," Solid-State Circuits, IEEE Journal of 15, 177-183 (1980).

${ }^{26}$ H. Ishikawa, H. Dobashi, T. Kodama, T. Furuhashi, and Y. Uchikawa, "Investigation of micro mechanical vibration of piezoelectric actuators using a stroboscopic sem," Journal of electron microscopy 42, 35-40 (1993).

${ }^{27}$ C.-L. Wong and W.-K. Wong, "In-plane motion characterization of mems resonators using stroboscopic scanning electron microscopy," Sensors and Actuators A: Physical 138, 167-178 (2007).

${ }^{28} \mathrm{M}$. Boudaoud, Caracterisation dynamique des bruits a lechelle nanometrique et commande robuste LPV de systemes de micromanipulation, Ph.D. thesis, Universite de Franche-Comte, Besancon, France (2012). 